\title{
कंठ साधना और संगीत शिक्षक
}

डॉ. नरेन्दर कौर

सह.आचार्य, संगीत विभाग गायन, आर.आर.एम.के. आर्य एम.एम.वी., पठानकोट

कंठ साधना से अभिप्राय अपनी आवाज़ पर नियन्त्रण पाना। कंठ साधना के द्वारा गायक अपने स्वर को इस प्रकार नियन्त्रित करता है कि उसकी आवाज़ में माधुर्य पैदा हो। चूँकि आवाज़ कम्पन से पैदा होती है, जब हवा हमारे कंट की स्वर तन्त्रियों से टकराती है तो हमारे गले से ध्वनि पैदा होती है, इस ध्वनि को संगीतोपयोगी बनाने के लिए कंठ-साधाना अत्यावश्यक है क्योंकि केवल कंठ-साधनाके द्वारा ही गायक अपने कंठ को दोष मुक्त कर सकता है एंव आवाज़ में सुन्दरता ला सकता है। प. ओम्कार नाथ ठाकुर कंठ साधना के बारे में कहते थे, "मेरा बाल कंठ अति मधुर था और तीनों सप्तकों में सुविधा से घूमता था किन्तु किशोरावस्था के आगमन के साथ ही मेरा कंठ फूटे मटके के समान फूट गया। गुरू आज्ञानुसार ब्रह्मा मुहूर्त में तानपूरा लेकर मैने स्वर-साधना की । आज यदि मेरे कंठ में कुछ है तो वह उस साधना का ही परिणाम है।"

जिस प्रकार एक खिलाड़ी के लिए शारीरिक व्यायाम की आवश्यकता होती है, ठीक उसी प्रकार एक गायक को कंठ साधना की आवश्यकता होती है। भले ही यह सत्य है कि सुरीली एवं मधुर आवाज़ प्राकृतिक देन है लेकिन इसके साथ यह भी सत्य है कंठ साधना के द्वारा साधारण आवाज़ को भी सुरीला और मधुर बनाया जा सकता है। हमारे सामने ऐसे कई उदाहरण है जिनके द्वारा हमें यह ज्ञात होता है कि कंठ साधना के अभ्यास से गले के कई दोषों को दूर किया जा सकता है। बड़े गुलाम अली खाँ, पं. विष्णु दिगम्बर पलुस्कर, पं. ओंकार नाथ ठाकुर, खुदाबख्श घग्गे, बहरे वहीद खाँ, पं. विनायक राव पटवर्धन इत्यादि कुछ ऐसे गायक रहे हैं जिन्होने कंट साधना के द्वारा अपने कंट को मधुर बनाया। निःसंदेह आवाज़ एक प्राकृतिक देन है किन्तु क्या एक पहलवान को कसरत की कोई आवश्यकता नहीं? क्या एक खिलाड़ी को अभ्यास की कोई आवश्यकता नहीं ? जिस प्रकार किसी भी खेल के अभ्यास से पहले शरीर को दौड़ लगाकर गर्म करते हैं जिसे हम Warmup कहते हैं उसी प्रकार किसी भी प्रकार का गायन हो स्वर अभ्यास की आवश्यकता तो अवश्य पड़ती है। यदि ध्वनि सम्बन्धी सभी बातों का ध्यान रखें तो हम अपनी आवाज़ की मधुरता को कायम रख सकते हैं और उसे और मधुर बना सकते हैं।

कंठ साधना से तात्पर्य है कंठ संस्कार जिसमें स्वर की साधना,श्वास पर नियन्त्रण, अच्छी श्रवण शक्ति का विकास, गले की माँसपेशियों का व्यायाम, जिह्टा का व्यायाम इत्यादि आते हैं।

स्वर साधना

यह साधना सुबह अथवा सांयकाल या फिर तब जबकि आपका कंठ विश्रामावस्था में हो, की जा सकती है। स्वर साधना के लिए कोई एक खास अभ्यास ही नहीं अपितु कई अभ्यास बताए जाते हैं, जिन्हें कंट विशेष के दोषानुसार अपनाया जा सकता है। कहने का तात्पर्य यह है कि आवाज़ के कुछ खास दोषों को दूर करने के लिए अथवा किसी खास स्वर या व्यंजन के निकास में यदि कोई दोष है तो उसी प्रकार का स्वर अभ्यास किया जाए जिससेवह दोष दूर हो जाए। संगीत का प्रभाव और उसमें रस की उत्पत्तिबहुत कुछ मधुर आवाज़ पर ही निर्भर करती है। बिना मधुर स्वर के संगीत का रसास्वादन नहीं किया जा सकता। मधुर स्वर के विषय में स्वामी हरिदास ने कहा है, "स्वर में जादू पैदा करो ताकि पशु पक्षी भी मग्न हो जाएं।" तानसेन ने कहा है,"प्रथम स्वर साध 
फिर बैठ गुनियन में रस की करे फुहार।" पं. ओम्कारनाथ ठाकुर ने सच्चे स्वर के बारे में कहा है, "स्वर के प्रभाव से मैने हिंसक शेर को भी मुग्ध कर दिया था और उसकी आँखों से पालतू जानवर जैसी मोहब्बत टपकने लगी थी।" मधुर स्वर में अपार शक्ति होती है। लता मंगेश्कर कहती हैं कि जहाँ संगीत सुरीला होता है वहाँ मैं अपने आप को खो देती हूँ।यह तो निशचित ही है कि यदि उचित मार्गदर्शन में उचित तरह से स्वर साधना की जाए तो वांछित कंट प्राप्त किया जा सकता है। मानवी कंठ सीमितताओं को ध्यान से रखते हुए ही स्वर साधना के निम्नलिखित अभ्यास अपनाए जा सकते हैं :-

1 षडज का अभ्यास :- कहते हैं कि 'एकहि साधह सब मिलै'। 'स' के अभ्यास से सातों स्वरों का लगाव ठीक हो जाता है। श्रीमति किशोरी अमोनकर जी का कहना है उन्होने एक-एक स्वर पर घण्टों अभ्यास किया। केवल वे ही नही अपितु सभी बड़े-बड़े उस्ताद जैसे अब्दुल करीम खाँ, रशीद खाँ, पंडित विष्णु दिगम्बर पलुस्कर, पं. ओंकारनाथ ठाकुर इत्यादि सभी 'स' के अभ्यास पर बल देते हैं। श्री लक्ष्मीनाराण गर्ग अपनी पुस्तक 'आवाज़ सुरीली कैसे करें' में लिखते हैं कि नाद साधना एक उच्चकोटि की साधना है और यह प्रत्येक उच्चकोटि के संगीतज्ञ के लिए आवश्यक है क्योंकि नाद की दृढ़ता के साथ-साथ दिव्य ज्ञान और आत्म शुद्धि के लिए यही एक सरल तथा शास्त्रोक्त साधना है। यदि नित्यप्रति इसकी साधना की जाए तो इससे इतने लाभ होते हैं कि उनको लेखनी द्वारा व्यक्त नहीं किया जा सकता। (लक्ष्मीनारायण गर्ग 69:1972)

2 प्रातः काल अथवा सांयकाल मन्द्र अभ्यास :- इसमें मन्द्र सप्तक के स्वरों पर जहाँ तक गला आसानी से जा सके वहाँ तक एक-एक स्वर पर पूर्ण श्वास भर अभ्यास किया जाए और कम्पन पर नियन्त्रण किया जाए।

3 प्रातःकाल तानपूरा लेकर अभ्यास के लिए बैठ जाएँ। यदि तानपूरा सुर में करना न आता हो तो आजकल इलैक्टोनिक तानपूराआता है जिसका प्रयोग किया जा सकता है अथवा तानपूरे के जोड़ी के तारो में से किसी एक को अपने साधारण स्वर पर मिला लें। यदि किसी भी तरह तानपूरा उपलब्ध नहीं तो अच्छे हारमोनियम का प्रयोग किया जा सकता है। थोड़ी देर तक तानपूरे को छेड़ते रहें और सुर सुनते रहें तत्पश्चात् प्रवाहित स्वर में मुँह बन्द करके नाक के स्वर में स्वर मिलाइए। जब पूर्णतया सुर मिल जाए तो उसे एक साँस में अधिक से अधिक देर तक टिकाने की कोशिश करें तथा ध्यान रखें कि स्वर विचलित न हो और स्थिर हो जाए। इस अभ्यास में यह ध्यान रखें कि साँस उतनी ही खींचे जितनी आसानी से सम्भव हो ताकि आवाज़ में किसी प्रकार का कम्पन अथवा गले पर कोई ज़ोर न पड़े एवं स्वर की स्वाभविकता बनी रहे। इसके बारे में श्री लक्ष्मीनारायण गर्ग लिखते हैं," आराम और आसानी से जितना लंबा स्वर खिंच सके उतना ही खींचिए ताकि स्वाभाविक स्वर गले में अपना एक रास्ता कायम कर ले। ठीक उसी प्रकार जिस प्रकार कि रेतीली जमीन या बालू पर पानी डाल देने से कुछ दूर बह कर वह सूख जाता है और अपनी एक दरार बना लेता है, तो उस जगह पर यदि दोबारा पानी डाला जाए तो वह उसी दरार से होकर बहता है, इधर-उधर नहीं जाता। इसी प्रकार आपका स्वर दृढ़ स्तम्भ के अनुसार हो जाएगा, उसकी मधुरता इधर-उधर फैलने की बजाए एकत्रित हो जाएगी तथा मंच पर प्रदर्शन के समय षडज लगते ही लोग स्वर सागर में डूबकर खो जाएँगे।" 
4 'स' स्वर पर नाक से गुँजन करने के पश्चात् रे ग म प ध नी स स्वरों पर दो दो मिनट तक गुंजन करें फिर इसी प्रकार अवरोह करते हुए वापिस आ जाए।

5 थोड़ी देर विश्राम के पश्चात मध्य स पर कू शब्द का उच्चारण करें। मध्य स पर जब आवाज़ साफ लगे तो एकदम पंचम पर कूशब्द लगाए तत्पश्चात् तार 'स' पर 'कू' का उच्चारण करने से आवाज़ में सफाई आती है। इसी प्रकार 'ई ऊ ए ओ' शब्दों के साथ भी यह अभ्यास गले में सफाई लाता है। सम्पूर्ण अभ्यास में इस बात का ध्यान अवश्य रखना चाहिए कि सम्पूर्ण अभ्यास स्वाभाविक रूप से किया जाए, गले पर किसी प्रकार का ज़ोर न डाला जाए। बलपूर्वक अभ्यास लाभ की बजाए हानि प्रदान करता है।(lbid.)

6 कुछ देर लगभग दो-तीन मिनट के लिए नासिका का अभ्यास कुछ इस प्रकार करें कि एक नासिका पर उंगली रखकर उसे बन्द कर लें और मुख भी बन्द रखकर ध्वनि निकालें इस दौरान दूसरी नासिका को उंगली से कोमलता के साथ जल्दी-जल्दी थपथपाए। इसी प्रकार अब दूसरी नासिका से भी यही अभ्यास करें। इससे श्लेष्मा इिल्ली स्पन्दित होगी और आवाज़ में गुँजन सा पैदा होगा।(ibid.)

7 आवाज़ निकालते समय उसकी गति तालु के ऊपरी भाग की ओर होनी चाहिए और गले पर अनावश्यक दबाव कभी नहीं डालना चाहिए, अन्यथा श्वास के स्वच्छन्द आवागमन में बाधा पड़ती है और वोकल कॉर्डस पूरी तरह अपना काम नहीं कर पाते। (वसन्त, 1999:614)

प्राणयाम एवं श्वास के अभ्यास

1 प्राणायाम के कुछ अभ्यासस्वर पर नियंत्रण पाने तथा फेफड़ों को मजबूत बनाने के लिए अति लाभदायक रहते हैं। श्वास हमारे स्वर को उत्पन्न करने का प्रमुख साधन है। स्वर यंत्र को उचित मात्रा में श्वास मिलने पर ही अच्छे स्वर अथवा अच्छी ध्वनि का निकास सम्भव है। इसके साथ ही यह भी आवयश्क है कि श्वास की पर्याप्त मात्रा समान गति से प्रवाहित हो तभी आवाज़ की मधुरता कायम रह पाएगी। श्वास अभ्यास का एक बहुत प्रभावशाली तरीका श्री लक्ष्मीनारायण गर्ग अपनी पुस्तक 'आवाज़ सुरीली कैसे करें में लिखते हैं- "धीरे-धीरे गहरी साँस लेकर फेफडों को पूरी तरह भर लीजिए। पसलियों के निचले हिस्से पर उँगली रखने पर पता चल जाएगा कि हवा भरने पर सीना फूल जाता है और डायाफ्राम (वक्ष और उदर के बीच की पेशी) की स्थिति में परिवर्तन आ जाता है। साधारण अवस्था में यह बाहर की ओर गोलाकार होती है किन्तु पसलियों के फुलाने पर यह सपाट हो जाती है। यह बात ध्यान में रखनी चाहिए कि साँस लेते समय सीने के ऊपरी हिस्से तथा कंधे तन न जाएँ अर्थात कंधो की स्थिति में अन्तर न आए। यदि बार बार ऐसा होता है तो इसका मतलब है कि निचली पसलियाँ ठीक से नहीं फैल रही हैं। जहाँ तक स्वर या आवाज़ का सम्बन्ध है, ऊपर के सीने में भरी जाने वाली आवाज़ कोई लाभ नहीं पहुँचाती। जब फेफड़े पूरी तरह फूल जाएँ तो धीरे धीरे साँस छोड़िए और फेफड़ों के खाली होने के साथ साथ सीने का गिरना अनुभव कीजिए। प्रणायाम करते समय इस बात का ध्यान अवश्य रखना चाहिए कि माँसपेशियों में किसी भी प्रकार का तनाव या खिंचाव पैदा न हो यदि ऐकसा हो तो थोड़ा "विश्राम लें और नि:श्वास छोड़िए।( गर्ग लक्ष्मीनारायण, 1972:60) 
2 तेज़ी से साँस लेकर उसे धीरे-धीरे छोड़ने से भी अच्छा श्वास नियंत्रण हो जाता है। इस अभ्यास में यह बात ध्यान रखनी चाहिए कि श्वास लेते समय आवाज़ बिल्कुल नहीं आनी चाहिए। (वसन्त, 1999:614)

3 शुद्ध वायु वाले वातावरण में मुँह फुलाकर एकदम से इकट्ठी वायु भरकर मुँह जल्दी से बन्द कर लें तथा थोड़ी देर के लिए रोके फिर एकदम से मुँह खोल दें सारी हवा एक दबाव(pressure) के साथ बाहर निकालें। इस अभ्यास से गले के साधारण खिंच खिंच या खराश ठीक होगी और गला साफ हो जाएगा।

4 श्री लक्ष्मीनारायण गर्ग अपनी पुस्तक आवाज़ सुरीली कैसे करें में लिखते हैं कि सुरीले गायन के लिए श्वास की गति और दिशा पर पूरा पूरा अधिकार होना चाहिए अन्यथा श्वास ग्रसनिका(pharynx) में जमा हो जाती है जहाँ वह कोमल तन्तुओं में बहुत-कुछ ध्वनियों को खो बैठती है। यदि आवाज़ को संतोषजनक बनाना है तो नासिका प्रदेश का पूरा पूरा इस्तेमाल आवश्यक है। स्वर को बनाने बिगाड़ने में इसका बहुत बड़ा हाथ रहता है। यह हड्डी से निर्मित होता है जिस पर हल्की-सी झिल्ली चढ़ी होती है। अतएव यह आवाज़ को साफ और चमकदार बना देता है। श्वास की दिशा इस प्रकार होनी चाहिए कि तालु के ऊपरी भाग का पूरा पूरा प्रयोग किया जा सके। श्वास लेने की क्रिया में तीन प्रमुख बातें एक दूसरे पर आधारित हैं(वसन्त, 1972:65):-

1 आवश्यक मात्रा में श्वास की सप्लाई

2 समान संयमित गति से श्वास का छोड़ा जाना।

3 तालु के ऊपरी भाग की ओर गति।

सही स्वर स्केल का चुनाव

कंठ साधना बिना किसी व्यवधान के हो इसके लिए सही स्केल का चुनाव अत्यावश्यक है। मध्य स का चुनाव ऐसा हो कि मन्द्र पंचम से तार मध्यम तक आवाज़ आसानी से और बिना किसी विकार के लग सके। स्वर साधना की सफलता सही स्केल के चुनाव पर निर्भर करती है। (S.S Awasthi,2017)

भोजन तथा परहेज़

अच्छा स्वास्थ्य अच्छे कंठ के लिए अति आवश्यक है। यदि शरीर तंदुरुस्त रहेगा तो ही कंठ भी सुरक्षित और सुदृढ़ रह सकेगा। अच्छे कंठ के लिए सन्तुलित आहार अत्यावश्यक है। गायक को हमेशा ध्यान में रखना चाहिए भोजन कि मुलायम और शक्तिवर्धक हो। अधिक गर्म या अधिक ठंडा भोजन नहीं करना चाहिए। तेज़ मसालेदार चटपटे भोज्य पदार्थ भी निषिद्ध हैं। तेज मिर्च, खटाई, आचार, तली एवं ठंडी वस्तुएं स्वर को बिगाड़ देती हैं। गायक को शुद्ध घी एवं शुद्ध तेल का ही प्रयोग करना चाहिए। माँस और नशीले पदार्थों का सेवन भी गायक के लिए वर्ज्य माना गया है। माँस में जीव के विषैले पदार्थ भी आ जाते हैं जो मानव शरीर को नुकसान पहुँचते हैं। इसके अतिरिक्त माँस का स्वर पर प्रभाव जानने के लिए हम पंछियों का उदाहरण ले सकते हैं। कोयल, तोता, मैना, कबूतर, मोर और पपीहा आदि अधिकांश धान्य और फलों का भोजन करते देखे जाते हैं और इनका स्वर अच्छा माना जाता हैं। इसके विपरीत कौवा,उल्लू, चील, गिद्ध आदि मांसाहारी होने 
से इनका स्वर अच्छा नहीं होता। अच्छे स्वर के लिए पके फल और सब्जियाँ बहुत फायदेमंद हैं। सूखे फलों से परहेज़ रखना चाहिए क्योंकि ये हमारे पाचन रस को सोखते हैं। इसी प्रकार नशीले पदार्थ भी क्षणिक जोश भले लाते हों लेकिन बाद में शिथिलता ही लाते हैं और अन्त में नशे के बगैर स्वर में कर्कशता और कम्पन पैदा हो जाता है। देखा जाता है कुछ गायक नशे के बिना गा ही नहीं सकते क्योंकि उन्हे नशे की ऐसी आदत पड़ जाती है कि नशे के बिना उनका स्वर लग ही नहीं पाता जिसके कारण वे नशा नहीं छोड़ पाते और धीरे-धीरे नशा उनकी मजबूरी बनता चला जाता है। उनका नशा उनके स्वर की प्राकृतिक शक्ति को नष्ट कर डालता है।(गर्ग लक्ष्मीनारायण, 1972:106)

\section{सुलभ औषधियाँ एवं इलाज}

जहाँ एक ओर कंठ को सुरीला बनाने के लिए विभिन्न प्रकार के अभ्यासों की आवश्यकता है वहीं कंठ को स्वस्थ्य रखने के लिए कुछ सुलभ औषधियों की जानकारी रखना भी एक गायक के लिए उतना ही आवश्यक है। कभी यदि अचानक सर्दी के कारण या गाते गाते गला बैठ जाए तो इस अवस्था में कुछ होम्योपैथिक दवाईयाँ काफी असरदार होती हैं। इसके लिए आर्जेटिम नाइटिकम का प्रयोग करना चाहिए। इसके अतिरिक्त कंट सुरीला बनाने के लिए कास्टिकम का प्रयोग भी लाभदायक रहता है। इसके अतिरिक्त मंच पर जाने से पूर्व यदि घबराहट हो, जिसके कारण पेट पर भी दबाव पड़ता है तो ऐसी स्थिति में होम्योपैथी की 'जेलसिमियम' अथवा 'सेनेगा' दवाई का प्रयोग करना चाहिए।

देसी औषधियों में कंठ सुधारक और योगी कंठिका अत्यंत उपयोगी दवाईयाँ हैं। शहद में अदरक के रस की बूँदे डालकर सेवन करने पर भी साधारण खाँसी जुकाम में राहत मिलती है। नित्य सुबह गला साफ रखने के लिए साफ पानी अथवा खाँसी जुकाम में नमक वाले पानी से गरारे करने पर भी कंठ साफ रहता है।

ज्यादा बोलने या गाने से अथवा जुकाम-सर्दी से यदि गला बैठ जाए तो एलोपैथिक दवाईयाँ जैसे 'टिंक्चर बेंजॉयन', 'यूकेलिप्टिस आयल' और मैनफल(पीपरमेंट) पानी में डालकर उसकी भाप लेने से आराम मिलता है। साथ ही गले पर 'मैंडिल्स थ्रोटपेन्ट', 'फैराइग्लिसरीन' तथा 'टैनिक एसिड ग्लिसरीन' का प्रयोग भी करें।गले कीसूजन व दर्द आदि दूर करने के लिए थोड़ी सी अजवायन पानी में उबालकर उसमें नमक मिलाकर गरारे करें।पानी में थोड़ी फिटकरी या डिसप्रिन की गोली डालकर गरारे करने पर भी फायदा होता है। (गर्ग लक्ष्मीनारायण, 1972:145)

श्रवण शक्ति का विकास - अच्छा श्रोता होना गायक का प्रथम गुण है। हमारा स्वर लगाव, हमारा कंट माधुर्य, हमारा गायन बहुत कुछ इस बात पर निर्भर करता है कि हम अपने गुरू की गायकी को कितना ध्यान से सुनते हैं। यही नहीं अपितु हमारे सम्पर्क में आने वाले योग्य गायक को सुनकर भी हम अपने गायन को बनाते हैं। जितनी बारीकी के साथ हम किसी कुशल गायक की गायकी को सुनते हैं उतना ही प्रभाव हम अपनी आवाज़ का बनाने में सक्षम होते हैं। इसलिए हमारे कंठ का सुरीलापन हमारी अच्छी श्रवण शक्ति पर भी निर्भर करता हैं। योग्य कलाकार के स्वर लगाव एवं ध्वनि गुण को हमें बहुत ध्यान से सुनना चाहिए और यथासम्भव अपने कंट को सुरीला बनाने का प्रयास करना चाहिए। 
कंठ साधना के लाभ

कंठ साधना के द्वारा जहाँ स्वर सुरीला होता वहाँ दूसरी ओर कई बीमारियाँ भी ठीक हो जाती हैं। कंठ साधना द्वारा मुख्यतः निम्नलिखित लाभ प्राप्त होते हैं :-

1 स्वर में तारता का विकास होता है। आप जहाँ चाहें आवाज़ लगा सकते हैं।

2 कंठ साधना से आवाज़ पर नियन्त्रण प्राप्त होता है। जिससे आवाज़ में ठहराव आता है।

3 स्वर अथवा वर्ण के लगाव में कई व्यक्तिगत दोष दूर हो जाते हैं।

4 आवाज़ में मधुरता एवं सुरीलापन बढ़ता है।

5 आवाज़ में गमक एवं हरकतों का विकास होता है।

6 श्वास पर नियन्त्रण पाने से कंठ सम्बन्धित कई बीमारीयाँ भी ठीक होती हैं। फेफडों को बल मिलता है। श्वास की बीमारी से भी राहत मिलती है। थायरॉयड जैसी बीमारी में भी कंठ साधना के द्वारा लाभ प्राप्त हो सकता है। लेखिका को स्वयं थायरायड की बीमारी थी जिसे आपने कंठ साधना द्वारा पूर्णरूप से ठीक किया है। इसके अतिरिक्त हृदय पर भी बल पड़ने से हृदय भी मजबूत हो जाता है। नाभि पर जोर पड़ने से पेट की कई बीमारियों से निज़ात पाया जा सकता है।

भारतीय एवंपाश्चात्यदृष्टिकोण

संगीत एक प्रयोगशील विषय भी है और कला भी। कलाकार को एक अच्छे प्रस्तुतिकरण के लिए कंठ-साधना की अनवरत आवश्यकता पड़ती रहती है। कंठ साधना एक प्रकार की तपस्या है जिसके बल पर गायक ऐसा आत्मविश्वास अर्जित कर लेता है कि न्यूनतम परिश्रम व ध्यान केन्द्रित करते हुए, स्वर व राग के समूह को इच्छानुसार प्रस्तुत कर सकता है। (कान्ता प्रसाद मिश्रा-2011:14)

वैदिक साहित्य पर यदि दृष्टिपात करें तो हम देखते हैं कि मैत्रेप्युपनिषद् एक सामवेदीय उपनिषद् है जिसमें गायत्री मन्त्र की गान आधारित व्याख्या व उपासना अत्यंत सरल और स्पष्टता से दी गई है। मैत्रायणी संहिता में संगीत विषयक विषय तथा विभिन्न प्रकार की वीणा जैसे वाद्यों का वर्णन है। श्रीमद्भागवत पुराण में हम मैत्रेय मुनि को एक महान वैज्ञानिक केरूप में पाते हैं। ऐसे मैत्रेय मुनि का नामोल्लेख हमें सप्तपदी के लेखक (अज्ञातविद्वान) द्वारा मिलता है जो कंठ संस्कार(voice culture) की व्याख्या करते हुए लिखते है :-

अशक्त गान बोधानां मूढ़ानामपि संमतम्।

प्रोक्तः मैत्रेयमुनिनेयं सप्तपदी मुच्यते। 3 ।

वस्तुतः सप्तपदी न पुराणं नो कलेति च ।

गूढ़विद्योदयेनेयं सरहस्यं प्रकाश्यते $|4|$

अर्थात् जिन्हे गायन का बोध नहीं है ऐसे मंदबुद्धि अनाड़ी के लिए मैत्रेयमुनि द्वारा मान्य 'सप्तपदी' प्रस्तुत है। सप्तपदी न तो कोई पुराण है न कला अपितु यह (संगीत जैसी) गूढ़ विद्या के रहस्यों पर प्रकाश डालती है। इसमें स्पष्ट किया गया है कि संगीत में प्रगति करने के लिए श्रुति एवं वर्ण दोनों का ज्ञान आवश्यक है उदाहरणार्थ 
द्वे विद्ये वदितव्ये श्रुतिज्ञानं तथाक्षरम्।

श्रुतिवर्णादिनिष्णातिर्गानं संपद्यते सदा $|8|$

पाश्चात्य देशों में गायकों ने वॉयस कल्चर तकनीक के द्वारा आवाज़ को सही रूप में ढालकर अपने गायन कौशल को एक आदर्श स्तर तक पहुँचाने में सफलता प्राप्त की है। हिन्दुस्तानी संगीत समाज वॉयस कल्चर जैसे विषय को किसी भी प्रकार का विशिष्ट दर्जा देने में सदैव उदासीन रवैया अपनाता रहा है जबकि पाश्चात्य देशों के गायक समाज ने वॉयस कल्वर को एक प्रतिष्ठित स्थान प्रदान किया है। वे समझते हैं कि "Voice culture is a branch of knowledge which deals with the right voice production techniques" अर्थात वायस कल्चर(कंठ संस्कार) वह विद्या है जो गले से सही ध्वनि उत्पादन की तकनीक को दर्शाती है। वॉयस कल्वर भारतीय संगीत जगत में एक नवीन विषय के रूप में देखा जा रहा है। हिन्दुस्तानी संगीत जगत में अभी तक तो यह भी निश्चित नहीं है कि वॉयस कल्वर विषय का प्रारूप किस प्रकार का होना चाहिए? इसके क्षेत्र में किन किन उप-विषयों का समावेश होना चाहिए (मिश्रा कान्ता प्रसाद, 2011:123)

यदि हम कंठसंस्कार शब्द को हमारे प्राचीन साहित्य में खोजने का प्रयत्न करें तो हम देखते हैं कि इस विषय पर कोई भी विस्तृत अथवा एकल ग्रन्थ अथवा पुस्तक हमें प्राप्त नहीं होती परन्तु इस विषय सम्बन्धी जानकारी हमें सम्बन्धित ग्रन्थों तथा पुस्तकों में कहीं-न-कहीं अवश्य प्राप्त हो जाती है। उदाहरणार्थ, शारंगदेव कृत 'संगीत रत्नाकर', भरत के 'नाटय शास्त्र' नारद कृत 'नारदीय शिक्षा' एवं 'संगीत मकरंद' तथा नान्य गोपाल की 'भरत भाष्यम्' में गान के गुणों एवं दोषों पर किए गये विवरण से हमें कंठ संस्कार की जानकारी अपरोक्ष रूप में प्राप्त हो जाती है। भरतमुनि ने नाट्यशास्त्र में कंट के छ: गुण माने है - श्रावक, घन, स्निग्ध, मधुर, अवधानवान् तथा त्रिस्थानशोभी-

"श्रावको(णो) थ घन: स्निग्धो मधुरो ह्यवधानवान्।

त्रिस्थानशोभीत्येवं तु षट् कण्ठस्य गुणा मता :।"

भरतमुनि ने छ: गुणों एवं पाँच दोषों का विवरण दिया है जबकि नारदीय शिक्षा में दस गुण एवं चौदह दोष एवं रत्नाकर में लगभग बाईस गुण एवं पच्चीस दोषों का उल्लेख मिलता है। नारदीय शिक्षा में तो प्रत्येक स्वर की उत्पत्ति का ढंग बताया गया है। केवल षडज की उत्पत्तिके लिए हमारी नासिका, कंठ, हृदय, तालु, जिह्टा और दाँतो का प्रयोग होता है।

$$
\text { नासाकण्ठमुरस्तालु जिह्टांदन्ताश्च संश्रितः षडतभि संजायते।। }
$$

वैदकि संगीत में स्वरों को संस्कार देने और कंठतन्त्री को विकसित करने के लिए मन्त्रों से पूर्व हिकार, हुंकार, ओम् आदि का उच्चारण आवश्यक माना गया। संगीत शिक्षा में भिन्न प्रकार के अलंकार या पल्टों का अभ्यास, "षडज स्वर की साधना" कंठ संस्कार के निमित ही है। प्राणायाम में ओंकार ध्वनि का उच्चारण करते समय निर्देशक नाभि से उदर, उदर से वक्ष और वक्ष से मस्तिषक के समस्त भाग को आन्दोलित करवाते हुए ही मुख से ध्वनि निकालने का निर्देश करता है। ओंकार ध्वनि का उठाव ही नाभि से होता है। जैसा कि भारतीय दर्शन में उल्लेख मिलता है, अग्नि और वायु के संयोग से ध्वनि उत्पन्न होती है। अर्थात् उदर की अग्नि से प्रेरित होकर वायु नाभि का स्पर्श करते हुए जब कंठ की तत्त्रियों पर आघात करती है तब कंठ से ध्वनि उत्पन्न होती है। 
प्रसिद्ध गायक बैजू बावरा की लिखी इस बन्दिश में भी उन्होने कंठ साधना की युक्ति पर बल दिया-

स रे रे ग म प ध नी सप्त सुर मो मन में ऐसो ही आवै......

स नी ध प स रे ग म कण्ठ बरन बनावै।

कहै बैजू बावरे, सुनिए गोपाल नायक,

संगीत मुद्रा सुद्ध बानी तंत्र मत सों बतावै।"

पं. विष्णु नारायण भातखण्डें अपनी पुस्तक हि.सं पं. क्रमिक पुस्तक मालिका भाग छः में लिखते हैं, "यह ठीक है कि मधुर आवाज़ ईश्वरीय देन है परंतु गायन के लिए बिल्कुल ही निरुपयोगी आवाज़ बहुत कम मनुष्यों में पाई जाती है। 95 प्रतिशत लोगों की आवाज़ गायन के योग्य बनाई जा सकती है। साधारण आवाज़ भी अभ्यास द्वारा सुरीली बनाई जा सकती है। इस महत्वपूर्ण विषय की ओर हमारे यहाँ काफी उदासीनता दिखाई देती है। आवाज़ अच्छी बनाने के लिए मुख्यतः तीन बातों की आवश्यकता होती है :-

1 आवाज़ ठीक प्रकार से निकालना

2 आवाज़की स्थिरता

3 श्वास नियन्त्रण।(वी.एन.भातखण्डे, 1973:24)

भले ही भारतीय विद्वानों शारंगदेव इत्यादि ने गायक के गुण दोषों का वर्णन तो किया है परन्तु उन गुणों को कैसे हासिल किया जाए एवं दोषो से कैसे मुक्ति पाई जाए इसका कोई उल्लेखे नहीं है। इसी प्रकार हि.सं.प. क्रमिक पुस्तक मालिका भाग छ: में भी आवाज़ को ठीक प्रकार से निकालने, र्थिर करने तथा श्वास पर नियन्त्रण पाने की विधि का कहीं उल्लेख नहीं मिलता।

भातखण्डे संगीत शास्त्र में गले की तैयारी के लिए सरगम के अभ्यास पर बल दिया गया है। बिलावल थाट के स्वरों के अभ्यास के साथ अन्य थाटों के स्वरों के आरोह-अवरोह के अभ्यास का भी निर्देश है। यह भी बताया गया है कि अच्छे स्वर का आरोहवरोह करते हुए धीरे धीरे लय भी बढ़ानी चाहिए जिससे गला अच्छा तैयार हो जाता है।(वी.एन.भातखण्डे, 1999:446)

पाश्चात्य विद्वानों ने शरीर विज्ञान, भौतिक विज्ञान एवं चिकित्सा शास्त्र के आधार पर कंठ साधना (voice culture) की प्रक्रिया को वैज्ञानिक ढंग से सिद्ध किया है।

Douglas Stanley जो कनाडा के भौतिकी के जानकार है (Physicist), ने जीभ के नीचे रखने वाला एक यंत्र बनाया जिससे जीभ की माँसपेशियों में लचीलापन लाया जा सके ताकि जीभ के बोलने की गति बढ़ाई जा सके जो गायन में बहुत सहायक सिद्ध होती है। इसके अतिरिक्त गला भी पूरा खुलता है।(Stanley Douglas 1950:101)

स्टेनली लिखते हैं, "गायक को जबड़े एवं होठों को सही स्थिति में रखना चाहिए। होंठ इस प्रकार से खुलें कि ऊपर एवं नीचे के दाँत दिखें, सिर एकदम सीधा हो आगे पीछे की ओर गिरा हुआ न हो। (Stanley Douglas 1950:112)

स्टेनली आवाज़ के अच्छे निकास के लिए कई प्रकार के manipulations बताते हैं, जैसे Hyoid Bone Manipulation, Thyroid Manipulation, Manipulation for Mixed Registration, Jaw Manipulation आदि। निस्संदेह ये सभी तरीके गले की माँसपेशियों को लचीला बनाने वाले हैं 
जिससे ध्वनि का निकास उचित होता है और ध्वनि अच्छी निकलती है। परन्तु किसी जतंपदमत की सहायता एवं सम्पूर्ण जानकारी के बिना हानिकारक भी सिद्ध हो सकता है। स्टेनली के शिष्य कॉरनिलियस रीड (Cornelius Reid) स्टेनली से बड़े प्रभावति थे लेकिन उनका मानना है कि स्टेनली के कड़े प्रतिबन्धों के कारण उनकी आवाज़ उल्टा खराब हो गई थी। यह बात उन्होने अपने Doctor of Musicial Arts ds dissertation में लिखी है।(Cornelius Reid, 2014:3)

Oakland Lyric Opera के म्यूज़िक डायरैक्टर Mark Narins अपने देश के महान गायकों द्वारा बहुत प्रभावित थे। वे अपनी आवाज़ अच्छी बनाना चाहते थे एवं गाना चाहते थे। उन्होने 39 वर्ष की आयु में Mr. Claude Heater से अपनी आवाज़ बनाने (voice culture) की training आरम्भ की। उस समय वे क्लारनेट, टोम्बो एवं पियानो बजाते थे।

लेकिन उनके trainerMr Clande ने उन्हें Stanley की तकनीक से अवगत करवाया और बताया कि साधारण आवाज़ को भी प्रभावशाली बनाया जा सकता है। उन्होने बताया मुँह खोलकर गाना एवं डायाफ्राम (diaphragm) से श्वास भरने से आवाज़ को support मिलता है एवं आवाज़ अच्छी बनती है। इस तरह अभ्यास करके Narins अच्छी आवाज़विकसित करने में सफल हुए। (www.oocities.org/great voice accessed on 4/9/2016)

कंठ साधना की सफलता के लिए यह जानकारी भी आवश्यक है कि हमारे कंठ से ध्वनि उत्पन्न कैसे होती है। हमारे कंठ के कौन से आन्तरिक अंग इसमें शामिल हैं? मानवीय ध्वनि में तीन अंग मुख्यतः शामिल रहते हैं स्पन्दन (The Vibrartor), गुंजन (The Resonator) तथा गति देने वाले अंग(The Articulator) जिन्हें पैदा करने के लिए हमारा श्वसन तंत्र (फेफड़े, डायाफ्राम और पेट की माँसपेशियाँ), लारिंक्स (vocal folds, कार्टिलेज, गले की माँसपेशियाँ और नाड़ियाँ) और सुपराग्लोटिक टैक्ट(Supraglotic tract, वोकल कॉर्ड का ऊपरी भाग, गले का पिछला हिस्सा, मुख, नासिका और नासिका का ऊपरी भाग अर्थात sinus cavaties) ये सभी शरीरिक अंग हिस्सा लेते हैं। कंठ ध्वनि उत्पन्न करने के लिए निम्नलिखित अंग भाग लेते हैं-

- Larnyx (voice box)

- Pharnyx

- Trachea

- Esophagus

- Spinal Column

- Diaphragm

- Vocal folds or vocal chords.

\begin{abstract}
${ }^{1}$ Frederick Husler and Yvonne Rodd writes in their book "Singing: The Physical Nature of the Vocal Organ", Singing not speaking is the nature of the voice. So voice engineering is a process of unlocking the innate abilities in a voice existing by its very anatomical and physiological nature. The anatomical areas which are relevant to the working of the singing voice are the breathing apparatus-abdominal, thoracic and clavicular breathing. The suspension system of the larynx-the web of muscles which holds the larynx in place from four different directions and the implications of the consequent positioning of the voice box and the freedom of movement of the vocal chords. The vibrating mechanism of the vocal chords-the chords and the membranes, their individual and conjuct working and the implications on register, timbre, sheen of the voice etc. The resonating systemincludes abdomen, chest, throat and head resonances.
\end{abstract}


स्वर यंत्र (The Larynx) स्वर यंत्र कार्टिलेज का बना एक डिब्बा है, जो श्वास नली के ठीक ऊपर स्थित होता है। इसमें तीन कार्टिलेज और दो वोकल कार्ड्स होते हैं। ये तीनों कार्टिलेज क्रमशः थायरॉयड, क्रीकॉयड और ऐरिटेनायड हैं। ऐरिटेनायड कार्टिलेज भी संख्या में दो होती हैं तथा वोकल कॉर्ड एक सिरे पर इनसे जुड़े रहते हैं। इनका दूसरा सिरा थायरॉयड कार्टिलेज के साथ मजबूती से जुड़ा रहता है। स्वर यंत्र थायरॉयड कार्टिलेज द्वारा पूरी तरह ढका रहता है इस कार्टिलेज के नीचे अँगूठी के आकार की क्रीकायड कार्टिलेज स्थित है। सामने की ओर क्रीको थायरॉयड माँसपेशी द्वारा यहा थायरॉयड कार्टिलेज से जुड़ी रहती है तथा आवश्यकतानुसार इन्ही माँसपेशियों की सहायता से थायरायड कार्टिलेज के पास पहुँच जाती है। थायराड कार्टिलेज और क्रीकॉयडकार्टिलेज के बने डिब्बेनुमा स्थान के भीतर पीछे की ओर दोनों एरिटेनॉयड कार्टिलेज स्थित हैं। वोकल कॉर्डस इन्ही के साथ जुड़े रहते हैं। दोनों एरिटेनायड कार्टिलेज के मध्य में एरिटेनॉयड माँसपेशी है जो थायरो-एरिटेनायड की विरोधी पेशी है। एरिटेनॉयड माँसपेशी के खिंचाव के कारण वोकल कार्डस खिंचते है और करीब आ जाते हैं। वोकल कार्डस के मध्य का भाग जो ग्लोटिस कहलाता है, इनके करीब आने पर बंद हो जाता है औरश्वास का अन्दर आना या बाहर जाना बन्द हो जाता है। इस प्रकार एक वाल्व(valve) का काम करता है। जब हम गाते हैं या बोलते हैं तो ग्लोटिस करीब करीब बन्द हो जाता है और वोकल कार्डस कुछ ऐसे सन्तुलन में खिंचे रहते है कि स्वर नियन्त्रण स्वतः ही हो जाता है। यहाँ पर ध्यान देने योग्य बात यह है कि वोकल कार्डस स्वयं कुछ नहीं करते। उनको गति देने वाली थायरो एरिटेनायड माँसपेशियाँ है। जो वोकल कॉर्डस में ही स्थित होती हैं तथा स्वर नियंत्रण में सर्वाधिक सहायक होती हैं।

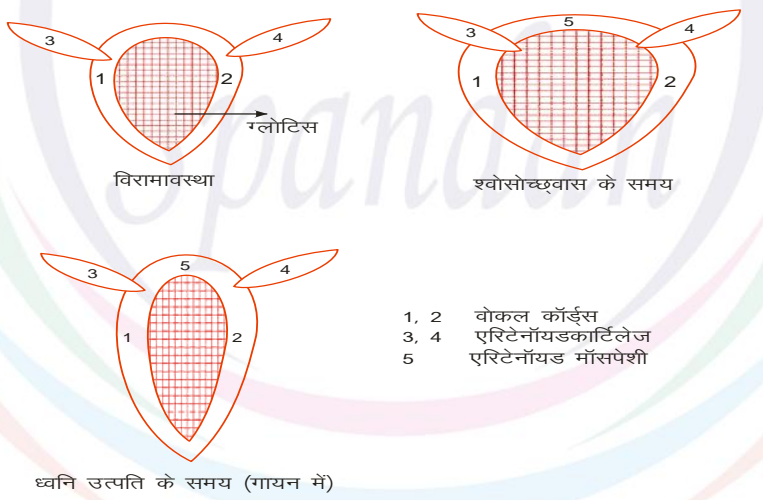

Fig. 1.Various positions of Glottis.

यदि ये माँसपेशियाँ गलत तनाव ले लें तो आवाज़ दोषपूर्ण निकलती है। यह गले के लिए भी हानिप्रद है। एरिटेनॉयड माँसपेशी के तनाव लेने पर वोकल कॉर्डस के खिंचने से ग्लोटिस बन्द हो जाता है तथा श्वास नली में से आने वाली वायु के दवाब से वोकल कार्डस आन्दोलित होते हैं और आवाज़ उत्पन्न होती है। (वसन्त, 1999: 605-606)

हमारे मुख से जो आवाज़ निकलती है उसमें एक प्रकार की गूँज होती है। डा. स्टेनले के अनुसार गूँजं ही सही आवाज़ के निर्माण का सबसे महत्वपूर्ण तत्त्व है। सृष्टि ने हमारे लिए कुछ गुंजन कक्ष बनाए है - 
मुख(Mouth)

नासा-विवर(Nasal Cavities)

खोपड़ी में स्थित खोखला भाग(head sinuses)

फेरिंक्स (Pharnyx)

जिनमें नेजेल(Nasal), ओरल(Oral) तथा लेरिंजियल फेरिंक्स(Laryngeal Pharynx) आते हैं।

जब वायु आन्दोलित होकर स्वर यंत्र से बाहर आती है तो वह नाक आदि से निकलती है और इसी कारण आवाज़ में गूँज होती है। जब फेरिंक्स कार्यरत होते हैं तो कंठ की संतलदहमंस बंअपजल तन जाती है। इस केवटी का आकार स्वर की ऊँचाई-नीचाई के अनुसार बदलता रहता है। गले का आकार जीभ के उद्गम पर आये हुए तनाव से महसूस किया जा सकता है। इस प्रकार गूँज लाने में सही रूप से जीभ की पेशियाँ सहायक होती है। इनके सुचारू कार्य से न केवल गुंजन कक्ष सही आकार में रहता है वरन् स्वर यन्त्र भी ठीक रहता है। कभी-कभी जब जीभ अत्यन्त कड़ी हो जाती है तो जो आवाज़ निकलती है उसे जीतवंजल अवपबम कहते हैं।(वसन्त, 1999:608)

वोकल कार्डस को नियन्त्रित करने वाले दो रजिस्टर माने गये हैं। डा. स्टेनले के अनुसार ये रजिस्टर हैं- 1 अपर रजिस्टर 2 लोअर रजिस्टर।

अपर रजिस्टर में एरिटेनॉयड माँसपेशी द्वारा लिए गये तनाव व उससे उत्पन्न आवाज़ का समावेश होता है जबकि लोअर रजिस्टर में थायरॉयड माँसपेशी के तनाव द्वारा उत्पन्न आवाज़शामिल है। निर्दोष स्वरात्पत्ति में दोनों ही रजिस्टरों का प्रयोग जरूरी है। स्थूल रूप से देखने पर मन्द्र सप्तक के स्वरों में लोअर रजिस्टर प्रबल होता है मध्य सप्तक के स्वरों में दोनों ही रजिस्टर बराबर रूप से कार्यरत रहते हैं और तार सप्तकमें अपर रजिस्टर पर अधिक भार आ जाता है।(वसन्त, 1999, 610)

डा. स्टेनले के अनुसार, गायक को सर्वप्रथम गायन आरम्भ करने से पहले यह स्रेच लेना चाहिए कि उसे क्या गाना है। फिर गुंजन कक्ष (मुख, नासिका, जिह्ञा) को आवश्यकतानुसार तैयार रखकर बिना किसी हिचकिचाहट के ग्लोटिस को धक्का पहुँचाए बिना स्वर लगाना चाहिए। फेरिंक्स के खोखले भाग (Pharnygeal Cavity) को तैयार करना, वोकल कॉर्ड का खिंचना तथा स्वर लगाना सब एक साथ होना चाहिए।

\section{विश्लेषण}

निस्संदेह अच्छी आवाज़ प्रकृति की देन है किन्तु उपयुक्त प्रशिक्षण के द्वारा सामान्य आवाज़ को भी अच्छा बनाया जा सकता है। यहाँ तक कि प्राकृतिक रूप से अच्छी आवाज़ होने पर भी प्रशिक्षण तो चाहिए ही, चाहे वो किसी योग्य शिक्षक अथवा गुरू से लिया जाए या स्वयं कोशिश की जाए । स्वयं कोशिश की बात मैं इसलिए कह रही हूँ क्योंकि हम अपने आस-पास कुछ ऐसे फिल्मी अथवा लोक गायक देखते हैं जिन्होने कभी किसी से नहीं सीखा ऐसे गायक प्राकृतिक आवाज़ एवं स्वयं अभ्यास करके अच्छा गाने लगते हैं। इस सन्दर्भ में सहगल, महेन्द्र कपूर का नाम लिया जा सकता है। यह तो निशचित है कि यदि हमें अपने बारे में जानकारी होती है, यदि हम अपनी कमज़ोरियों को समझ लेते हैं त्रे हम उन्हें दूर भी कर सकते हैं। आवाज़ को सुरीला बनाना इन्सान के अपने हाथ में ही होता है। डा. स्टेनले के अनुसार ष्ne who accidently falls on a correct technique 
has a good voice". तो आवश्यकता होती है सही ढंग से आवाज़ के लगाव की। यदि हम अपने स्वर यंत्र की पूर्ण जानकारी रखते हैं तो हम अपनी आवाज़ सही ढंग से लगा सकते हैं। अच्छी आवाज़ बनाना बिल्कुल वैसा ही है जैसा कि कच्चे माल से कोई उत्पाद तैयार करना। बेशक उत्पादन की विशेषता अथवा गुण कच्चे माल की क्वालिटी पर निर्भर करती है।किन्तु यह भी सत्य है कि यदि सामग्री अच्छी होने पर भी उसे सही ढंग से उपयोग न किया जाए तो भी प्रोडक्शन अच्छा नहीं बन सकता। दूसरी ओर यदि सामग्री सामान्य किस्म की भी हो लेकिन यदि उसका उपयोग सही ढंग और सजगता से किया जाए तो उत्पादन अच्छा बन सकता है। संगीत विशारद के लेखक वसन्त के अनुसार, "ऐसा तो कोई एकाध गायक ही होगा जिसका गला स्वभावतः निर्दोष हो"(वसन्त, 1999:611)। अतएव पाश्चायत्य देशों में आवाज़ के सम्बन्ध में कंठ की संरचना, श्वास की जानकारी अर्थात् Diaphragmic breathing, गुंजन(resonating) अंग इत्यादि की जानकारी सामान्य रूप से सर्व-विदित हो चुकी है। लेकिन हिन्दुस्तानी संगीतमें अभी तक ऐसी कोई वैज्ञानिक उपलब्धि नहीं हो सकी है। इस सन्दर्भ में श्री लक्ष्मीनारायण गर्ग द्वारा लिखी पुस्तक 'आवाज़ सुरीली कैसे करें बहुत अच्छा प्रयास है जिसमें बहुत ही अच्छे एवं प्रभावशाली अभ्यास एवं व्यायाम बताए गये हैं।

पाश्चात्य देशों में तो किसी भी प्रकार की आवाज़ को सुरीला बनाने के लिए वैज्ञानिक प्रयास किए जाते हैं और आवाज़ को पहचान कर बताया जाता है कि अमुक आवाज़ किस प्रकार की गायकी के लिए उपयुक्त रहेगी। जबकि हमारे देश में ऐसा कोई भी सिस्टम अभी तक विकसित नहीं किया गया है। यहाँ तक कि यहाँ की यूनीवर्सिटियों अथवा बड़े-बड़े संगीत विद्यालयों में भी अलग से कंठ-संस्कार की कोई सुविधा उपलब्ध नहीं है। कुछ दहाके से डा. उन्नीकृष्णन इस क्षेत्र में सराहनीय कार्य कर रहें हैं।

कंठ साधना का ज्ञान अच्छे शिक्षण में भी सहायक है क्योंकि गायन सीखने वाले सभी शिष्यों अथवा विद्यार्थियों की आवाज़ स्वाभाविकतया मधुर अथवा सुरीली नहीं होती एवं सभी शिष्यों को आवाज़ लगाने का ढंग सिखाने के लिए भी सही निर्देशन की आवश्यकता होती है। यदि शिक्षक अथवा गुरू स्वयं तो बहुत अच्छा गाता हो किन्तु वह शिष्य को सही दिशा निर्देशन न कर पाए तो शिक्षक होने के नाते वह अपना दायित्व सफलता पूर्वक नहीं निभा सकता। इसलिए किसी भी संगीत गुरु, उस्ताद अथवा शिक्षक के लिए तो कंठ साधना की जानकारी होना अत्यावश्यक है तभी वह आवाज़ लगाव का ढंग सिखा सकता है अन्यथा यह कार्य बिल्कुल भी सम्भव नहीं है।

भारतीय खासकर हिन्दुस्तानी संगीत में भी कंठ संस्कार के लिए ऐसा प्रावधान बना दिया जाए कि प्रत्येक वह चाहवान जो संगीत से प्रेम करता है और खुद गायन सीखना चाहता है, गायन सीख सके। खासकर यूनीवर्सिटियों में जहाँ संगीत विभाग है वहाँ कंठ संस्कार के ज्ञाता अथवा जतंपदमत की नियुक्ति अलग से हो अथवा जो भी गायन के शिक्षक हों उनके लिए कंठ साधना की विशेष जतंपदपदह का प्रबन्ध कर इसे अनिवार्य बनाया जाए ताकि वे आगे अपने विद्यार्थियों को इस दिशा में प्रशिक्षण दें और इस विषय का प्रचार हो सके प्रत्येक गायन संगीत के शिक्षकों के लिए वॉयस कल्चर का वर्कशाप अनिवार्य रूप से रखा जाए। इस दिशा में यूनीवर्सिटीस के संगीत विभाग अहम् भूमिका निभा सकते हैं। 


\section{सन्दर्भ ग्रन्थ सूची}

लक्ष्मीनारायण गर्ग - आवाज़ सुरीली कैसे करें? संगीत कार्यालय हाथरस,1972।

कान्ता प्रसाद मिश्रा - 'वॉयसकल्चर : ज्ञान एवं परंपरा' कनिष्क पब्लिशर्स, नई दिल्ली, 2011।

महारानी शर्मा(2012) संगीत मणि, श्री भुवनेश्वरी प्रकाशन, इलाहाबाद।

वी.एन. भातखण्ड(1973)-हि.स. प. क्रमिक पुस्तक मालिका भाग-6 संगीत कार्यालय हाथरस।

वी.एन. भातखण्डे (1957)-हि.सं. प. भातखण्डे संगीत शास्त्र, संगीत कार्यालय हाथरस।

वसन्त (1999) - संगीत विशारद 23तक मकपजपवदए संगीत कार्यालय हाथरस।

Douglas Stanley(1950)- "Voice : Applied Science of Vocal Art".New York, Pitman www.oocities.org/great voice/accessed on 4/9/16.

Reid Cornilius L. (2014) 'Voice Building Exercises', Dissertation prepared for the degree of 'Doctor of Musical Arts', University of North Texas.

S.A.K. Durga (2007) Voice Culture : The art of voice cultivation, B.R. Rhythms Delhi

Manjiree Gokhale (2011) Voice Tantra Yoga Mantra : Harmony of Inner of Expressed voice, Power Publishers, Kolkata.

Prof. Frederick Husler, and Yvonne Rodd (1976) 'Singing : The Physical Nature of the vocal organ' Hutchinson \& Co. Ltd, Surrey U.K.

Music Thoery, Wikipedia, the free encyclopediaen, wikipedia. org/wiki/Music_theory.

S.S. Awasthi, A critique of Hindustani Music and Music Eucation, Dhanpat Rai \& Sons, JalandharNew Delhi

Whitefield Ward(1881)- The Throat in its relation to Singing. C.A. Welles. 